Supporting Information:

\title{
From Thin Films to Nanopillars: Tunable Morphology of Covellite via Radio Frequency Magnetron Sputtering for Cost-Effective Photothermal Vaporization
}

Ng Zhen Quan, Cavin ${ }^{\dagger}{ }^{\ddagger}$,, Ashutosh Rath ${ }^{\ddagger}$, Andrew T.S. Wee ${ }^{\dagger}$ §, Stephen J. Pennycook ${ }^{\ddagger}$ and Daniel H. C. Chua, ${ }^{*}, \neq$

${ }^{+}$NUS Graduate School for Integrative Sciences and Engineering, National University of Singapore, 119077, Singapore

‡ Department of Materials Science and Engineering, National University of Singapore, 117575, Singapore

$\S$ Department of Physics, National University of Singapore, 117551, Singapore

Keywords: CuS Nanopillars, Photothermal Vaporization, RF Magnetron Sputtering

*Corresponding Author: danielchua@,nus.edu.sg 


\section{TABLE OF CONTENT}

Figure S1: Layout and Schemetic Diagram of the Photothermal Vaporiztion Test

Figure S2: X-ray Photoelectron Spectroscopy Broadscan of CuS Thin Film and Nanopillars.

Figure S3: Bandgap Measurement via Tauc Plot and Reflectance Measurements of CuS Thin Film and Nanopillars.

Figure S4: Observation of Over-etched CuS Nanopillars via Secondary Electron Microscope. 


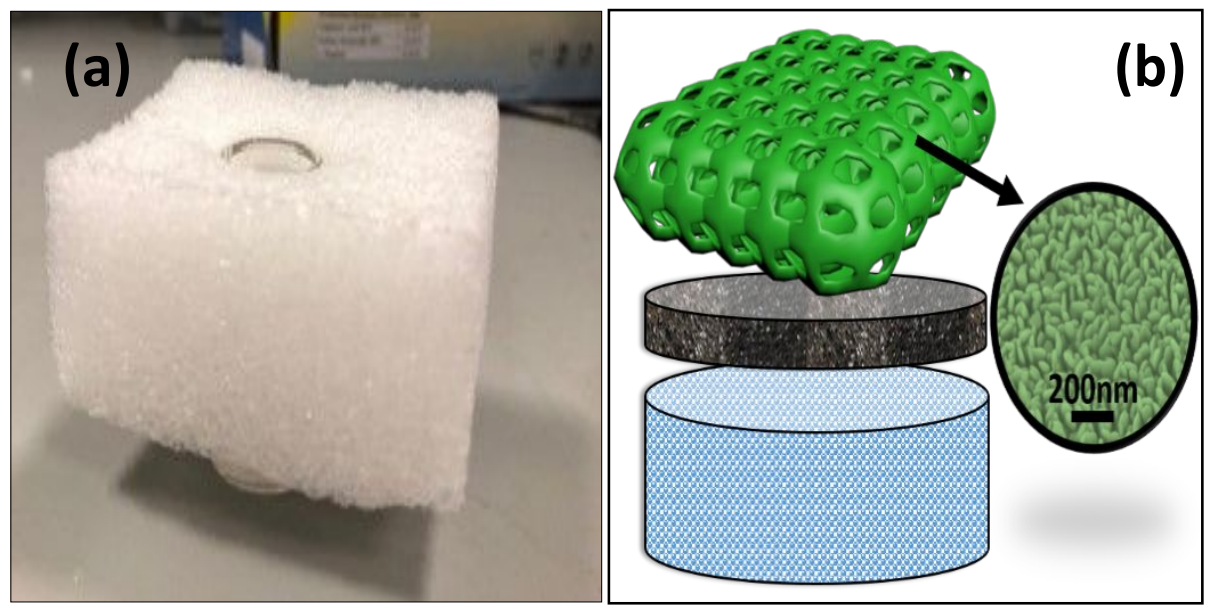

Figure S1: (a) Insulation used around the glass vial. (b) Schematic setup of the photothermal test. $10 \mathrm{ml}$ of water (blue) with polyethylene foam (black) to aid with flotation. Melamine foam coated with CuS nanopillars (green) at the top.
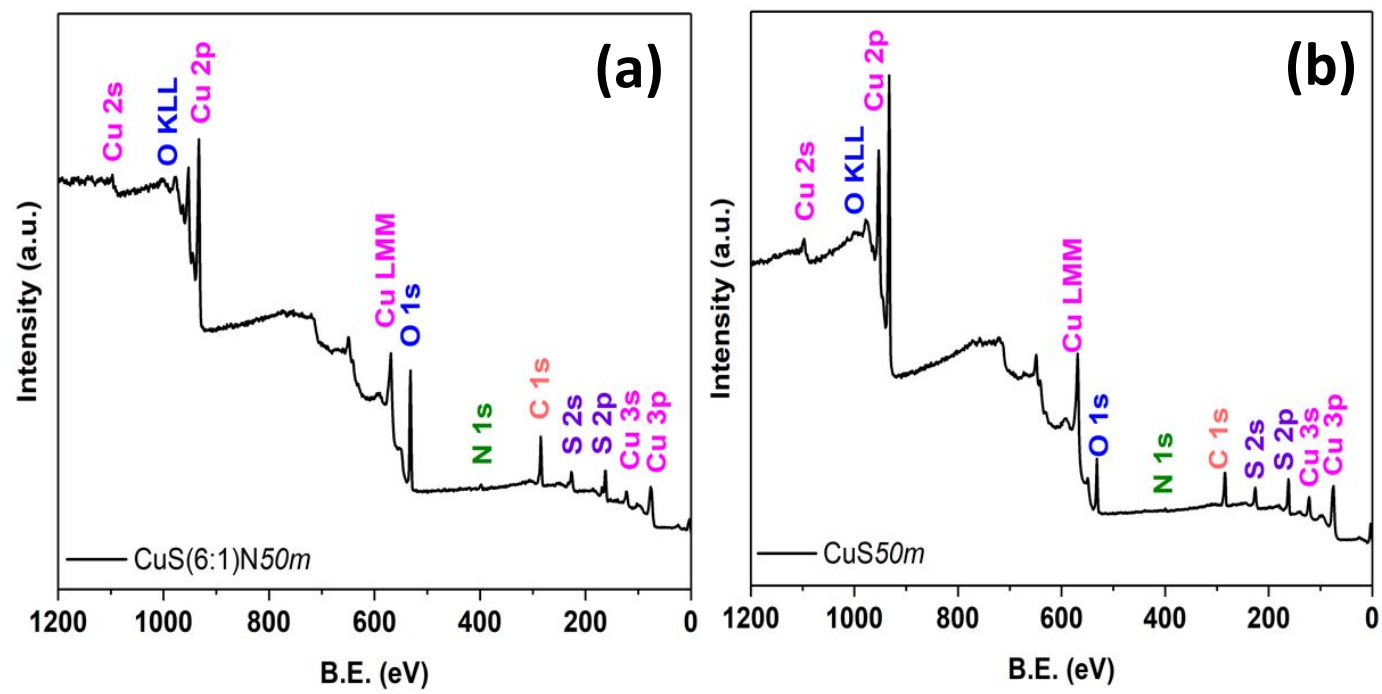

Figure S2: XPS survey scan of (a) $\mathrm{CuS}(6: 1) \mathrm{N} 50 m$ and (b) CuS50m. Nitrogen 1s peak is not prominent on both scans. 

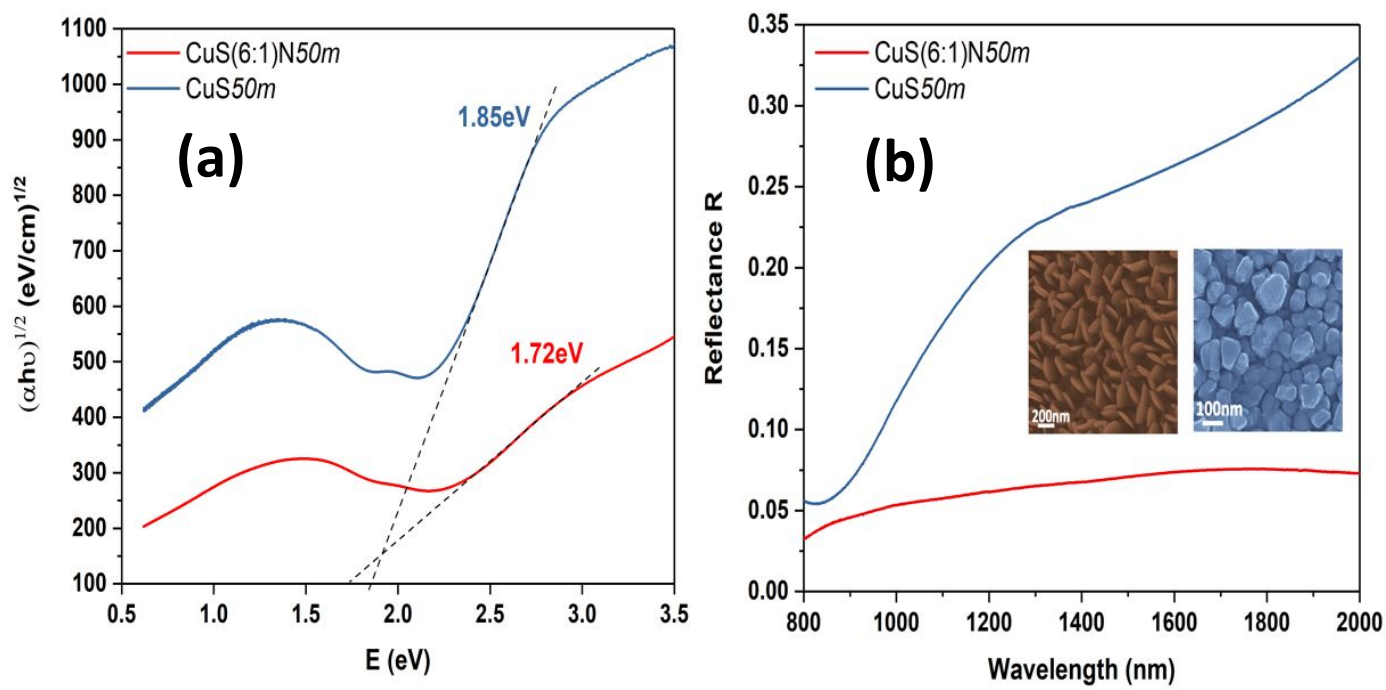

Figure S3: (a) Tauc Plot and (b) Reflectance of $\mathrm{Cu}(6: 1) \mathrm{N} 50 m$ and CuS50m

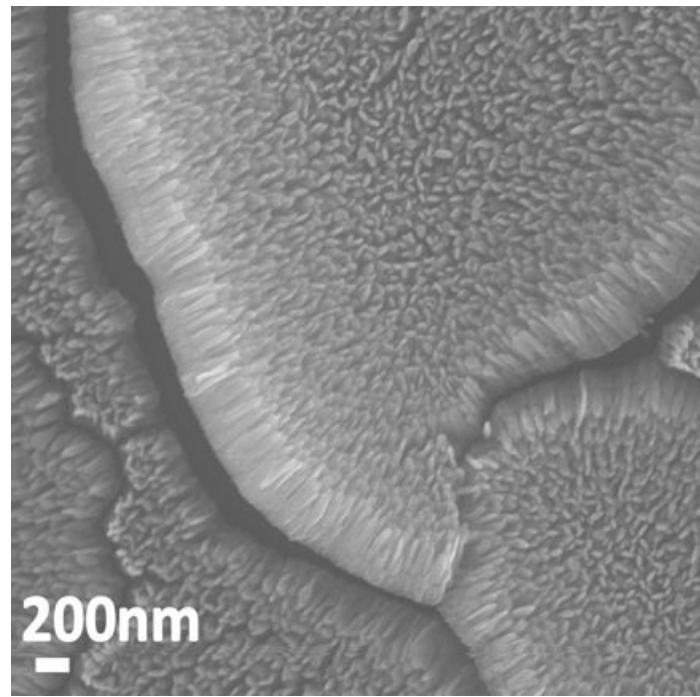

Figure S4: SEM image of CuS(3:1)N. Nanopillars delaminate easily from the substrate. 